\title{
Helicobacter pylori isolated from patients who later failed $H$. pylori eradication triple therapy readily develop resistance to clarithromycin
}

Correspondence
Intetsu Kobayashi
mbc-ka@sa2.so-net.ne.jp

Received 6 September 2005

Accepted 3 February 2006

\author{
Intetsu Kobayashi, ${ }^{1}$ Takeshi Saika, ${ }^{1}$ Hiroe Muraoka, ${ }^{1}$ Kazunari Murakami ${ }^{2}$ \\ and Toshio Fujioka ${ }^{2}$ \\ ${ }^{1}$ Chemotherapy Division, Mitsubishi Kagaku Bio-Clinical Laboratories, Inc., 3-30-1 Shimura, \\ Itabashi-ku, Tokyo 174-8555, Japan \\ ${ }^{2}$ Department of General Medicine, Faculty of Medicine, Oita University, Hasama-machi, \\ Oita 879-5593, Japan
}

\begin{abstract}
In this study, the ease of selection of clarithromycin resistance was investigated in clarithromycin-susceptible Helicobacter pylori strains isolated from patients with $H$. pylori infection prior to the administration of triple-combination eradication therapy (clarithromycin plus amoxicillin plus a proton pump inhibitor). Clarithromycin-susceptible strains isolated from ten patients in whom the eradication therapy was successful and from six patients in whom the eradication therapy was unsuccessful were exposed serially to subinhibitory concentrations of clarithromycin. The number of transfers required for the MICs of the strains to increase by 8- and 32-fold were 6.6 and 7.2, respectively, in the successful eradication group, and as few as 2.4 and $1 \cdot 5$, respectively, in the unsuccessful eradication group. The number of transfers required for the $\mathrm{A} 2142 \mathrm{G}$ or $\mathrm{A} 2143 \mathrm{G}$ point mutation of the 23S rRNA gene to be detected in the strains were 5 and 8 , respectively, for the strains in the successful eradication group, and 1 and 2 , respectively, for the strains in the unsuccessful eradication group. These results suggest that patients in the unsuccessful eradication group were infected with strains of $H$. pylori that readily became resistant to clarithromycin on exposure to the drug.
\end{abstract}

\section{INTRODUCTION}

According to the Gastroenterology Society of Japan guidelines for the eradication of Helicobacter pylori infection, the ideal eradication therapy for infected gastric or duodenal ulcer patients is a combination regimen consisting of clarithromycin plus amoxicillin plus a proton pump inhibitor. Eradication rates with this triple therapy have been reported to be as high as 80-90\% (European Helicobacter pylori Study Group, 1997), although they varied by medical institution (van den Hulst et al., 1996; Ducóns et al., 1999; Broutet et al., 2001). However, the current lack of an established secondline therapy for patients in whom eradication of clarithromycin-resistant $H$. pylori strains is unsuccessful is a serious issue. In addition, reports exist describing patients infected with clarithromycin-susceptible strains in whom $H$. pylori eradication was still unsuccessful (Ducóns et al., 1999); the mechanism for this failure remains unclear.

In the present study, we investigated the ease of induction of clarithromycin resistance in clarithromycin-susceptible strains of $H$. pylori isolated prior to the eradication therapy, both from patients in whom the eradication therapy was successful (the successful eradication group) and from those in whom it was ultimately unsuccessful (the unsuccessful eradication group).

\section{METHODS}

Bacterial strains. Clarithromycin-susceptible H. pylori strains were isolated from infected gastric ulcer or duodenal ulcer patients, as described previously (Suzuki et al., 1999), before the patients were treated with the triple-combination eradication therapy in accordance with the guidelines for the diagnosis and therapy of $H$. pylori infection (Japanese Society for Helicobacter Research, 2000). Sixteen clarithromycin-susceptible strains were used in the study, comprising ten strains isolated from patients in the successful eradication group and six from patients in the unsuccessful eradication group. The effect of eradication therapy (successful or unsuccessful) was determined according to the guidelines mentioned above.

Antibiotics and determination of antimicrobial susceptibility. Clarithromycin obtained from Taisho Pharmaceutical was used to select resistant mutants and for measurement of the MIC. The MIC of clarithromycin was determined by the agar dilution method, according to the guidelines established by the NCCLS (2003). Mueller-Hinton agar (Difco Laboratories) plates containing $5 \%$ sheep blood and serial 2-fold dilutions of clarithromycin were inoculated with $1-3 \mu \mathrm{l}$ of a saline suspension of the $H$. pylori isolates equivalent to a $2 \cdot 0 \mathrm{McF}$ arland standard $\left(1 \times 10^{7}-1 \times 10^{8}\right.$ c.f.u. $\left.\mathrm{ml}^{-1}\right)$. 
Plates were incubated for $72 \mathrm{~h}$ at $35^{\circ} \mathrm{C}$ in a microaerophilic atmosphere. The MIC was defined as the lowest concentration of clarithromycin that completely inhibited bacterial growth.

Selection of clarithromycin resistance. Clarithromycin-resistant mutants were generated by the serial-passage method. A $72 \mathrm{~h}$ growth of each strain on blood agar base no. 2 (Oxoid) supplemented with $5 \%$ defibrinated horse blood was inoculated using a swab onto the blood agar containing clarithromycin at a concentration of $0.5 \times$ MIC. The surface growth after $72 \mathrm{~h}$ incubation was subcultured onto medium containing the same and twice the previous concentration of clarithromycin. This procedure was repeated ten times serially, using the surface growth on medium containing progressively increasing concentrations of clarithromycin.

Statistical analysis. Statistical analysis was performed by Student's $t$-test. A statistically significant difference was defined as having a value of $P<0 \cdot 02$.

Detection of point mutations. Point mutations at A2142 or A2143 of the 23S rRNA gene were identified by the PCR-RFLP method described by Versalovic et al. (1997). Chromosomal DNA was isolated from approximately ten colonies of each strain by phenol/chloroform extraction and ethanol precipitation. PCR amplification of the domain $\mathrm{V}$ region of the $23 \mathrm{~S}$ rRNA gene was performed with the primers $5^{\prime}$-AGTCGGGACCTAAGGCGAG-3' and 5'-TTCCCGCTTAGATGCTTTCAG-3'. The conditions for amplification were as follows: pre-denaturation at $94{ }^{\circ} \mathrm{C}$ for $3 \mathrm{~min}$, followed by 40 cycles of denaturation at $94^{\circ} \mathrm{C}$ for $1 \mathrm{~min}$, annealing at $50^{\circ} \mathrm{C}$ for $2 \mathrm{~min}$ and extension at $72^{\circ} \mathrm{C}$ for $2 \mathrm{~min}$, with post-extension at $72{ }^{\circ} \mathrm{C}$ for $7 \mathrm{~min}$. For detection of the A2142G and A2143G mutations, $7 \cdot 5 \mathrm{U}$ MboII (Takara Shuzo) or $5 \mathrm{U}$ BsaI (New England Biolabs) were added to each PCR product and digestions were performed for $14 \mathrm{~h}$ at 55 or $37^{\circ} \mathrm{C}$, respectively. The digested PCR products were analysed by electrophoresis on a $1 \%$ agarose gel and stained with ethidium bromide.

\section{RESULTS}

\section{Clarithromycin MICs for the mutant and parent strains}

The number of transfers required to select resistance in the test strains obtained from patients in the successful and unsuccessful eradication groups and the respective MICs are shown in Table 1 . The clarithromycin MIC for all ten of the original strains (B1-B10) isolated from patients in the successful eradication group was $0 \cdot 015 \mu \mathrm{g} \mathrm{ml}^{-1}$. By the third transfer, the MIC for strain B4 had increased 8-fold compared with the original strain and thereafter increased a further 4-fold, but still remained comparatively low at $0.5 \mu \mathrm{g} \mathrm{ml}^{-1}$, even at the tenth transfer. In contrast, the MICs for strains B3 and B8 increased greatly at the fifth transfer and increased further to $128 \mu \mathrm{g} \mathrm{ml}^{-1}$ or more by the tenth transfer. In addition, the MIC for strain B9 increased at the eighth transfer and was $64 \mu \mathrm{g} \mathrm{ml}^{-1}$ at the tenth transfer. There were few changes in the MICs for the other strains, with MIC values of $\leqslant 0 \cdot 5 \mu \mathrm{g} \mathrm{ml}^{-1}$ by the tenth transfer.

The clarithromycin MICs for isolates obtained from patients in the unsuccessful eradication group ranged from 0.03 to $0.06 \mu \mathrm{g} \mathrm{ml}^{-1}$, which was 2- to 4 -fold higher than those for the isolates obtained from the patients in the successful eradication group. The MICs for all of the strains except R1 and R6 increased rapidly, as early as the first or second transfers, and reached $16 \mu \mathrm{g} \mathrm{ml}^{-1}$ or more by the second transfer; thus, the number of transfers required before resistance was selected was much smaller.

Table 1. Number of transfers required for induction of resistance in clarithromycin-susceptible strains and corresponding MICs in the successful and unsuccessful eradication groups

\begin{tabular}{|c|c|c|c|c|c|c|c|c|c|c|c|}
\hline Strain & $\begin{array}{c}\text { MIC of } \\
\text { original strain }\end{array}$ & \multicolumn{10}{|c|}{ MIC $\left(\mu \mathrm{g} \mathrm{ml}^{-1}\right)$ after the following number of transfers: } \\
\hline B1 & $0 \cdot 015$ & $0 \cdot 015$ & $0 \cdot 015$ & $0 \cdot 015$ & $0 \cdot 06$ & $0 \cdot 06$ & $0 \cdot 06$ & $0 \cdot 06$ & $0 \cdot 06$ & $0 \cdot 06$ & $0 \cdot 06$ \\
\hline B2 & $0 \cdot 015$ & $0 \cdot 03$ & $0 \cdot 06$ & $0 \cdot 03$ & $0 \cdot 03$ & $0 \cdot 03$ & $0 \cdot 03$ & $0 \cdot 03$ & $0 \cdot 06$ & $0 \cdot 06$ & $0 \cdot 12$ \\
\hline B3 & $0 \cdot 015$ & $0 \cdot 015$ & $0 \cdot 03$ & $0 \cdot 03$ & $0 \cdot 03$ & 16 & 16 & 16 & 32 & 128 & 128 \\
\hline B6 & $0 \cdot 015$ & $0 \cdot 03$ & $0 \cdot 03$ & $0 \cdot 03$ & $0 \cdot 03$ & $0 \cdot 06$ & $0 \cdot 06$ & 0.06 & $0 \cdot 12$ & $0 \cdot 25$ & $0 \cdot 25$ \\
\hline B7 & $0 \cdot 015$ & $0 \cdot 015$ & $0 \cdot 03$ & $0 \cdot 03$ & 0.03 & $0 \cdot 06$ & $0 \cdot 03$ & 0.06 & $0 \cdot 06$ & $0 \cdot 06$ & $0 \cdot 06$ \\
\hline B8 & $0 \cdot 015$ & $0 \cdot 015$ & $0 \cdot 06$ & $0 \cdot 03$ & $0 \cdot 06$ & 16 & 8 & 8 & 32 & 64 & $>128$ \\
\hline B9 & $0 \cdot 015$ & 0.015 & $0 \cdot 015$ & $0 \cdot 015$ & $0 \cdot 015$ & 0.03 & $0 \cdot 03$ & 0.06 & 8 & 16 & 64 \\
\hline B10 & $0 \cdot 015$ & $0 \cdot 015$ & $0 \cdot 03$ & 0.015 & 0.03 & 0.03 & $0 \cdot 25$ & $0 \cdot 25$ & $0 \cdot 25$ & $0 \cdot 5$ & $0 \cdot 5$ \\
\hline R3 & 0.06 & 64 & $>128$ & - & - & - & - & - & - & - & - \\
\hline R4 & 0.03 & 0.03 & 16 & 8 & 16 & 16 & 16 & 16 & 64 & 64 & - \\
\hline R5 & $0 \cdot 06$ & 8 & 16 & 8 & 8 & 16 & 64 & 64 & - & - & - \\
\hline R6 & $0 \cdot 03$ & 0.03 & $0 \cdot 03$ & 0.03 & 0.03 & $0 \cdot 03$ & 0.03 & $0 \cdot 03$ & $0 \cdot 03$ & 0.03 & 0.03 \\
\hline
\end{tabular}


Table 2. Comparison of the number of transfers required for acquisition of resistance by clarithromycin-resistance induction

\begin{tabular}{|c|c|c|c|c|c|c|}
\hline Group & $\begin{array}{l}\text { Strains } \\
\quad(n)\end{array}$ & $\begin{array}{l}\text { MIC of original } \\
\text { strains }\left(\mu \mathrm{g} \mathrm{ml}^{-1}\right)\end{array}$ & 8 -fold & 16-fold & 32-fold & 64-fold \\
\hline Unsuccessful eradication group & 6 & $0 \cdot 03-0 \cdot 06$ & $2 \cdot 4^{a} \dagger(5)$ & $2 \cdot 6^{b}(5)$ & $1 \cdot 5^{a}(4)$ & $1 \cdot 5(4)$ \\
\hline
\end{tabular}

${ }^{\star}$ Number of strains given in parentheses.

$\dagger$ Significantly different from the successful eradiation group at $P<0 \cdot 01$ (a) or $P<0.02(b)$.

\section{Mean number of transfers}

The mean number of transfers required for the MIC to increase by 8 -fold or more after the selection of resistance was compared between the isolates from the two groups of patients (Table 2). A much smaller number of transfers was required for the MICs to increase by 8-, 16-, 32- or 64fold for isolates obtained from patients in the unsuccessful eradication group.

\section{5 rRNA gene point mutation}

The presence and type of point mutations at each serial transfer in each strain isolated from patients in the successful and unsuccessful eradication groups after the selection of resistance, the number of transfers until detection of point mutations and the clarithromycin MICs of the strains at the time of appearance of the point mutations are shown in Table 3. Among the strains obtained from the patients in the successful eradication group, three strains showed point mutations after the selection of resistance and the clarithromycin MICs of these strains were 8 or $16 \mu \mathrm{g} \mathrm{ml}^{-1}$. Two of the strains (B8 and B9) had the A2143G mutation and one strain (B3) had the A2142G mutation after the fifth serial transfer or later, thus showing a correlation between the presence of mutations and increased MICs.

Among the strains obtained from the patients in the unsuccessful eradication group, four of the strains had point

Table 3. Point mutation type in the $23 S$ rRNA gene and corresponding clarithromycin MICs and number of transfers required for detection of mutations

\begin{tabular}{|c|c|c|c|c|}
\hline \multirow{2}{*}{ Strain } & \multicolumn{2}{|c|}{ Mutation } & \multirow{2}{*}{$\begin{array}{c}\mathrm{MIC} \\
\left(\mu \mathrm{g} \mathrm{ml}^{-1}\right)\end{array}$} & \multirow{2}{*}{$\begin{array}{l}\text { Number } \\
\text { of transfers }\end{array}$} \\
\hline & A2143G & A2142G & & \\
\hline \multicolumn{5}{|c|}{ Successful eradication group } \\
\hline B3 & - & + & 16 & 5 \\
\hline B8 & + & - & 16 & 5 \\
\hline B9 & + & - & 8 & 8 \\
\hline \multicolumn{5}{|c|}{ Unsuccessful eradication group } \\
\hline $\mathrm{R} 2$ & - & + & 128 & 2 \\
\hline R3 & - & + & 64 & 1 \\
\hline $\mathrm{R} 4$ & - & + & 16 & 2 \\
\hline R5 & + & - & 8 & 1 \\
\hline
\end{tabular}

mutations after the selection of resistance and these strains became resistant at the first or second transfer. Three of these strains (R2, R3 and R4) had the A2142G mutation with clarithromycin MICs of $\geqslant 16 \mu \mathrm{g} \mathrm{ml}^{-1}$. The remaining strain (R5) had the A2143G mutation and a clarithromycin MIC of $8 \mu \mathrm{g} \mathrm{ml}^{-1}$.

\section{DISCUSSION}

The reported eradication rates following triple-combination therapy vary with the medical institution and range widely from 70 to $95 \%$ (van den Hulst et al., 1996; Ducóns et al., 1999; Broutet et al., 2001). These differences among medical institutions may be attributable to the rate of resistance of $H$. pylori to clarithromycin during the early stage of eradication therapy. According to Murakami et al. (2002), the eradication rates following triple-combination therapy in patients infected with clarithromycin-resistant strains may be as low as $8 \%$ and extremely low compared with the typical eradication rate. However, the eradication rate of strains for which elevated MICs were observed, but which were still susceptible to amoxicillin, was not as low. These findings suggest that clarithromycin may play a major role in the eradication of $H$. pylori.

The mechanisms of resistance to macrolides, the antibiotic category to which clarithromycin belongs, are generally accepted to be: (i) inactivation of enzyme (hydrolysis and modification: ereA, ereB and phosphotransferase), (ii) low accumulation (active efflux and low membrane permeability: $\operatorname{erp} A$ and $m s r A$ ) and (iii) changes in the target regions (changes in rRNA: ermA mutation in the 23S rRNA gene), and vary greatly according to the bacterial species (TaitKamradt et al., 2000). Resistance of $H$. pylori to clarithromycin is usually due to mutation in the $23 \mathrm{~S}$ rRNA gene. Moreover, it has been reported that these organisms readily become resistant to clarithromycin when they are exposed to the drug at sub-MIC levels (Haas et al., 1990). In a previous study, we detected clarithromycin resistance at a high frequency after eradication therapy with a regimen containing clarithromycin (Kobayashi et al., 1996). It would be reasonable to assume that the resistant strains were selected during exposure to clarithromycin based on the evidence that susceptible strains were isolated before the treatment (Kim et al., 2003); furthermore, spontaneous resistance is rare in H. pylori (Taylor et al., 1997). In our previous study, 
strains isolated before eradication therapy that were obtained from patients in the unsuccessful eradication group were resistant to clarithromycin and had the A2143G or A2142G point mutation. Since these patients had no previous history of eradication therapy for $\mathrm{H}$. pylori, it is highly possible that the resistance had been selected previously in these $H$. pylori strains by macrolides given to the patients in the past.

In the present study, the development of resistance to the drug followed one of two patterns, i.e. the strains became resistant to clarithromycin after a small number of exposures or the strains became resistant to clarithromycin in a stepwise manner after long-term exposure to the drug. This suggests that there are strains that can readily develop resistance to clarithromycin following short-term exposure to the drug administered in vivo. The mechanism underlying such a rapid development of resistance to clarithromycin has not yet been clarified, but resistance of the strains to the drug is considered to be selected at an early stage due to the development of mutations in the originally susceptible strains. Fontana et al. (2002) have reported that the T2717C mutation of domain VI is present specifically in moderately resistant strains at a clarithromycin MIC of $1 \mu \mathrm{g} \mathrm{ml}{ }^{-1}$, which differs from the A2142G and A2143G mutations of domain $\mathrm{V}$ of the $23 \mathrm{~S}$ rRNA gene reported to date. No T2717C mutation was detected in the strains that readily became resistant to clarithromycin in our previous investigation (data not shown). In addition, in Japan, there are very few reports of strains developing resistance at a clarithromycin MIC of $1 \mu \mathrm{g} \mathrm{ml}^{-1}$ (Kobayashi et al., 2001) as shown by Fontana et al. (2002). Therefore, the mechanism underlying the rapid development of resistance in the strains in the present study is presumed to be different from that in the aforementioned strains.

The above results indicate that, among the clinically isolated H. pylori strains in this study, there were strains that readily became resistant, even after only a small number of transfers with exposure to clarithromycin at sub-MIC levels, and careful attention should be paid to eradication therapy with regimens containing mainly clarithromycin in patients infected with these strains. Further investigation is needed to determine the mechanism of such rapid development of resistance to clarithromycin in these strains, which would be the key to inhibiting the increase in the number of resistant organisms.

\section{REFERENCES}

Broutet, N., Marais, A., Lamouliatte, H., de Mascarel, A., Samoyeau, R., Salamon, R. \& Mégraud, F. (2001). cagA status and eradication treatment outcome of anti-Helicobacter pylori triple therapies in patients with nonulcer dyspepsia. J Clin Microbiol 39, 1319-1322.
Ducóns, J. A., Santolaria, S., Guirao, R., Ferrero, M., Montoro, M. \& Gomollón, F. (1999). Impact of clarithromycin resistance on the effectiveness of a regimen for Helicobacter pylori: a prospective study of 1-week lansoprazole, amoxicillin and clarithromycin in active peptic ulcer. Aliment Pharmacol Ther 13, 775-780.

European Helicobacter pylori Study Group (1997). Current European concepts in the management of Helicobacter pylori infection. The Maastricht Consensus Report. Gut 41, 8-13.

Fontana, C., Favaro, M., Minelli, S., Criscuolo, A. A., Pietroiusti, A., Galante, A. \& Favalli, C. (2002). New site of modification of $23 \mathrm{~S}$ rRNA associated with clarithromycin resistance of Helicobacter pylori clinical isolates. Antimicrob Agents Chemother 46, 3765-3769.

Haas, C. E., Nix, D. E. \& Schentag, J. J. (1990). In vitro selection of resistant Helicobacter pylori. Antimicrob Agents Chemother 34, 1637-1641.

Japanese Society for Helicobacter Research (2000). Guidelines for diagnosis and treatment of Helicobacter pylori infection. Jpn $J$ Helicobacter Res 2, 2-12 (in Japanese).

Kim, J. J., Kim, J. G. \& Kwon, D. H. (2003). Mixed-infection of antibiotic susceptible and resistant Helicobacter pylori isolates in a single patients and underestimation of antimicrobial susceptibility testing. Helicobacter 8, 202-206.

Kobayashi, I., Toda, H., Hasegawa, M., Nishida, M., Fujioka, T. \& Nasu, M. (1996). Drug susceptibility of Helicobacter pylori freshly isolated from gastrointestinal mucosa of patients with peptic ulcer. Jpn J Chemother 14, 719-722 (in Japanese with English summary).

Kobayashi, I., Saika, T., Muraoka, H., Inoue, M. \& Nasu, M. (2001). Relationship between clarithromycin breakpoint for Helicobacter pylori and point mutation in $23 \mathrm{~S}$ rRNA gene. Jpn J Chemother 46, 236-240 (in Japanese with English summary).

Murakami, K., Sato, R., Okimoto, T., Nasu, M., Fujioka, T., Kodama, M., Kagawa, T., Sato, S., Abe, H. \& Arita, T. (2002). Eradication rates of clarithromycin-resistant Helicobacter pylori using either rabeprazole or lansoprazole plus amoxicillin and clarithromycin. Aliment Pharmacol Ther 16, 1933-1938.

NCCLS (2003). Performance standards for antimicrobial susceptibility testing. Thirteenth informational supplement M100-S13. Wayne, PA: NCCLS.

Suzuki, J., Muraoka, H., Kobayashi, I., Fujita, T. \& Mine, T. (1999). Rare incidence of interspousal transmission of Helicobacter pylori in asymptomatic individuals in Japan. J Clin Microbiol 37, 4174-4176.

Tait-Kamradt, A., Davies, T., Cronan, M., Jacobs, M. R., Appelbaum, P. C. \& Sutcliffe, J. (2000). Mutations in $23 \mathrm{~S}$ rRNA and ribosomal protein L4 account for resistance in pneumococcal strains selected in vitro by macrolide passage. Antimicrob Agents Chemother 44, 2118-2125.

Taylor, D. E., Ge, Z., Purych, D., Lo, T. \& Hiratsuka, K. (1997). Cloning and sequence analysis of two copies of a $23 \mathrm{~S}$ rRNA gene from Helicobacter pylori and association of clarithromycin resistance with $23 S$ rRNA mutations. Antimicrob Agents Chemother 41, 2621-2628.

van den Hulst, R. W., Keller, J. J., Rauws, E. A. \& Tytgat, G. N. (1996). Treatment of Helicobacter pylori infection: a review of the world literature. Helicobacter 1, 6-19.

Versalovic, J., Osato, M. S., Spakovsky, K. \& 7 other authors (1997). Point mutations in the 23S rRNA gene of Helicobacter pylori associated with different levels of clarithromycin resistance. J Antimicrob Chemother 40, 283-286. 